\title{
Autofocus Imaging: Image reconstruction based on inverse scattering theory
}

\author{
Jyoti Behura ${ }^{1}$, Kees Wapenaar ${ }^{2}$, and Roel Snieder ${ }^{3}$
}

\begin{abstract}
Conventional imaging algorithms assume single scattering and therefore cannot image multiply scattered waves correctly. The multiply scattered events in the data are imaged at incorrect locations resulting in spurious subsurface structures and erroneous interpretation. This drawback of current migration/imaging algorithms is especially problematic for regions where illumination is poor (e.g., subsalt), in which the spurious events can mask true structure. Here we discuss an imaging technique that not only images primaries but also internal multiples accurately. Using only surfacereflection data and direct-arrivals, we generate the up- and down-going wavefields at every image point in the subsurface. An imaging condition is applied to these up- and downgoing wavefields directly to generate the image. Because the above algorithm is based on inverse-scattering theory, the reconstructed wavefields are accurate and contain multiply scattered energy in addition to the primary event. As corroborated by our synthetic examples, imaging of these multiply scattered energy helps eliminate spurious reflectors in the image. Other advantages of this imaging algorithm over existing imaging algorithms include more accurate amplitudes, target-oriented imaging, and a highly parallelizable algorithm.
\end{abstract}

\section{INTRODUCTION}

Subsalt images are commonly of poor quality because of insufficient illumination and the presence of surface-related and internal multiples generated from the overlying strong reflectors (salt interfaces, water bottom, etc.). The energy of multiply scattered events can be so strong that the true subsurface structure is masked, leading to incorrect interpretation. Surface-related multiples can be suppressed to a large extent (Verschuur et al., 1992); however, prediction of internal multiples is an extremely challenging task. Instead of eliminating the internal multiples from reflection data, it would be beneficial to image them as they might increase illumination crucial for subsalt imaging. Conventional imaging algorithms, including reverse-time migration (RTM), assume single scattering and therefore multiples in the data are imaged at incorrect positions, resulting in spurious subsurface structures. Here, we discuss an imaging algorithm that includes internal multiples in the imaging operator, in addition to primary reflections.

In 1D scattering theory, Marchenko's integral equation (Marchenko, 1955; Agranovich and Marchenko, 1963) determines the relation between the wavefield in the interior of a medium and the reflected impulse response. This equation was originally derived for spherically symmetric media (and therefore 1D) and uses only reflected waves. A significant breakthrough was made by Rose (2002b) who utilize an iterative scheme to solve the Marchenko equation in one dimension. This approach, which he named single-sided autofocusing, determines the wavefield in 1D media by focusing the incident wave at a specified time. The input to this algorithm consists of only the reflected waves recorded on one side of the experiment. Aktosun and Rose (2002) and Rose (2002a) prove that this incident wave that focuses the wavefield in the interior is in fact a delta function (band-limited in practice), followed by the time-reversed solution of the Marchenko equation. Broggini and Snieder (2012) show that summation of this incident wave and the resulting reflection response yields the Green's function between the focal point and the surface. Wapenaar et al. (2013) extend the 1D iterative algorithm of Rose (2002b) to 3D. Combining the methodology of Broggini and Snieder (2012) for Green's function retrieval, Wapenaar et al. (2013) propose a methodology for reconstructing the 3D impulse response for any virtual source

Manuscript received by the Editor 12 November 2013; revised manuscript received 14 January 2014; published online 25 April 2014.

${ }^{1}$ Formerly Colorado School of Mines, Department of Geophysics, Golden, Colorado, USA; presently Seismic Science LLC, Littleton, Colorado, USA. E-mail: jyotibehura@gmail.com.

${ }^{2}$ Delft University of Technology, Department of Geoscience and Engineering, Delft, Netherlands. E-mail: c.p.a.wapenaar@tudelft.nl.

${ }^{3}$ Colorado School of Mines, Department of Geophysics, Golden, Colorado, USA. E-mail: rsnieder@mines.edu.

(C) 2014 Society of Exploration Geophysicists. All rights reserved. 
in the subsurface using surface reflection data and the direct arrivals from the virtual source to the receivers on the surface.

Beside extending Rose's iterative algorithm to higher dimensions, Wapenaar et al. (2011) also show that the wavefield at any interior location can also be decomposed into the down-going and up-going wavefields at the virtual source. Here, we discuss an imaging technique, originally introduced by Wapenaar et al. (2011) and elaborated by Behura et al. (2012) as Newton-MarchenkoRose Imaging, that uses the up- and down-going wavefields directly to image the subsurface. We call the algorithm Autofocus Imaging because the wavefield extrapolation is done using the "autofocusing" scheme (Rose, 2002b; Wapenaar et al., 2013). Beside demonstrating our imaging technique on synthetic examples, we discuss its advantages and drawbacks over existing imaging methods, in particular RTM.

\section{ALGORITHM}

Any seismic imaging algorithm consists of two steps: wavefield extrapolation and imaging condition. For example, RTM is a twoway imaging technique that uses wavefields extrapolated in time by accurately implementing the wave equation in a background velocity model. Under this technique, the source- and receiver-wavefields are reconstructed by forward-propagating the source signature and back-propagating the receiver recordings using a numerical solution of the wave equation (commonly a finite-difference algorithm). Wavefield extrapolation is followed by the application of an imaging condition (commonly crosscorrelation or deconvolution, Claerbout [1985]) to image the reflectors. In simple terms, the imaging condition determines the similarity between the wavefield that is incident on the reflector and the wavefield that is reflected. The similarity is high at a reflector position and low elsewhere. Imaging using conventional algorithms, however, assumes that all multiples (surface-related and internal) have been suppressed from the data; if not, the multiples could show up as spurious reflectors in the image. If all multiples are suppressed in the data, conventional imaging algorithms produce the correct image of the subsurface (Figure 1a) provided one uses an accurate (and smooth) background velocity model. Multiples, if present, however, will result in spurious subsurface structure as demonstrated in Figure 1b.

Unlike RTM, Autofocus Imaging uses the up-going $\left(G^{-}\right)$and down-going $\left(G^{+}\right)$Green's functions to generate the image (Wapenaar et al., 2011). The $G^{-}$and $G^{+}$make up the full Green's function $G$, which is the impulse response on the surface for a source at the image point (Broggini and Snieder, 2012). The upgoing $G^{-}$is the impulse response at the surface receivers due to an upward-radiating virtual source at the image point, whereas $G^{+}$is the impulse response due to a downward-radiating virtual source (Wapenaar et al., 2011). Because the Marchenko equation is based on inverse scattering, the reconstructed up- and downgoing wavefields contain most multiply scattered energy. In the absence of a reflector (Figure 1c), the up- and down-going Green's functions do not coincide kinematically at the depth of the virtual source as illustrated in Figure 1c; i.e., $G^{-} \neq G^{+}$. On the other hand, in the presence of a reflector at the image point (Figure 1d), $G^{-} \propto r G^{+}$, where $r$ is the reflection coefficient. Note that although at the reflector location, $G^{-}$and $G^{+}$are kinematically equal, their amplitudes differ by the magnitude of the reflection coefficient (which can be recovered using a deconvolution imaging condition).

The synthetic example in Figure 2 demonstrates the above arguments. We generate $G^{-}$and $G^{+}$for two different depth locations in the model shown in Figure 2a. The shallower image point at $z=$ $375 \mathrm{~m}$ does not coincide with any reflectors, whereas the image point at $z=500 \mathrm{~m}$ does. Note that $G^{-}$and $G^{+}$for the image point at $z=375 \mathrm{~m}$ do not coincide (Figure 2b), but for $z=500 \mathrm{~m}$, they do (Figure 2c). Therefore, application of any imaging condition will result in a reflector at $z=500 \mathrm{~m}$ with contributions from the primary as well as internal multiples. In addition to the primary wavefield, most multiply scattered energy will also be imaged accurately using Autofocus Imaging. Note that the autofocusing algorithm is
Figure 1. Schematic of a standard imaging scheme in 1D showing the principle of image reconstruction in the (a) absence and (b) presence of internal multiples. Schematic in 1D showing the upgoing $\left(G^{-}\right.$, in red) and downgoing $\left(G^{+}\right.$, in blue) Green's functions for an image point (c) not on a reflector and (d) on a reflector. The black dot in (c) and (d) represents the point of focus, i.e., the virtual source. a)

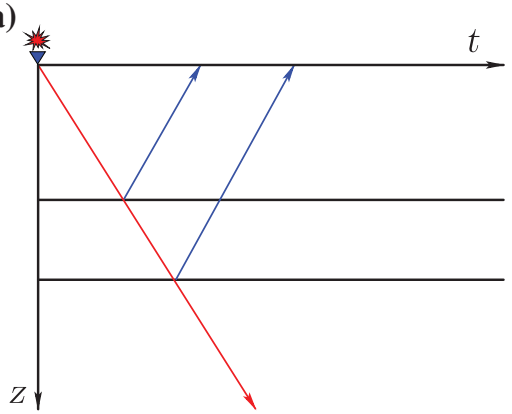

c)

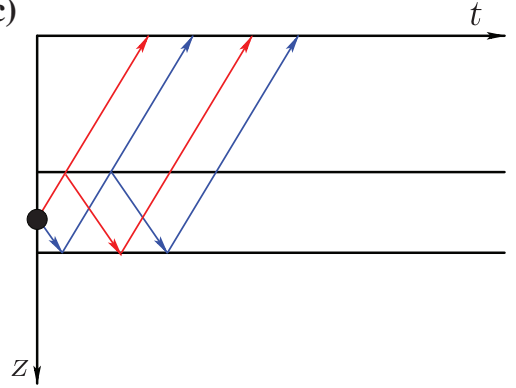

b)

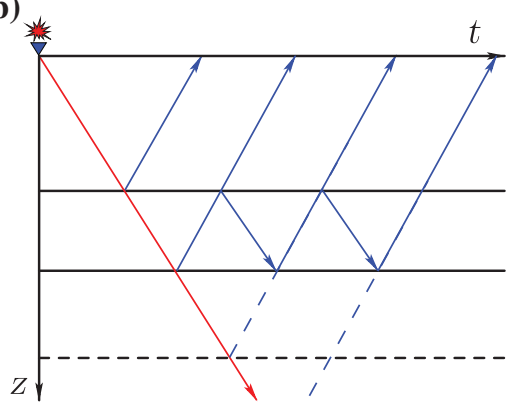

d)

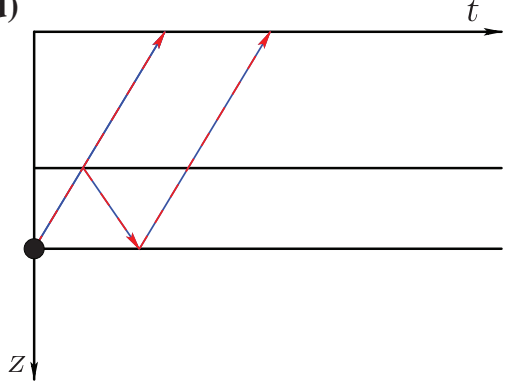


currently applicable only to the data containing internal multiples (and devoid of surface-related multiples). Therefore, the input data to Autofocus Imaging must have the surface-related multiples suppressed prior to imaging. The role of surface-multiples in autofocusing is currently under investigation.

The pseudocode for Autofocus Imaging is given by Algorithm 1.

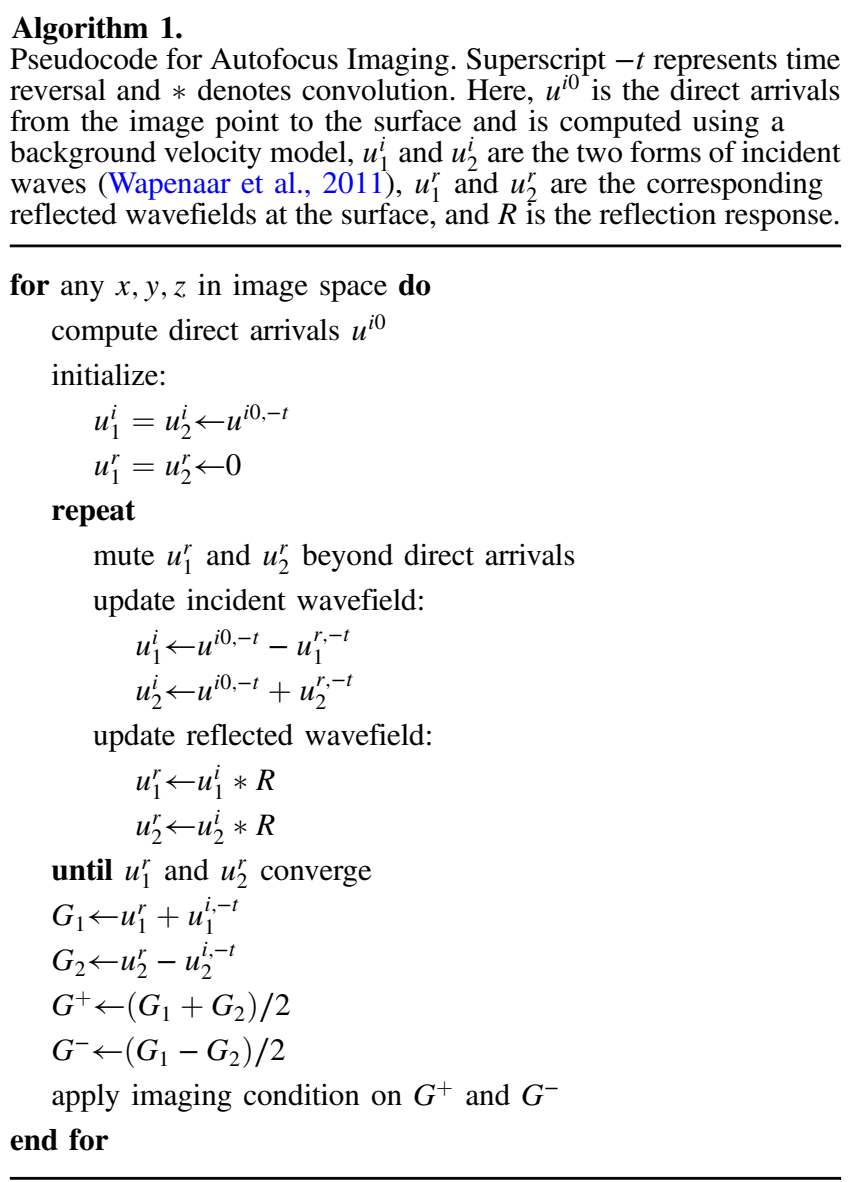

The reflection response $R$ is computed from surface reflection data by deconvolving the source signature from the recorded waves, and therefore, the knowledge of the source signature is critical for this algorithm. Moreover, as mentioned above, the input surface seismic data should be devoid of surface-related multiples and direct arrivals between the surface sources and receivers. $G_{1}$ and $G_{2}$ represent the full Green's functions between the focal point and the surface (Broggini and Snieder, 2012). Note that a background velocity model is necessary to compute the direct arrivals $u^{i 0}$ at the surface from an impulse at the image point. For the imaging condition, we use either the crosscorrelation or the deconvolution operation (Claerbout, 1985). Broggini et al. (2013) extend our imaging algorithm using multi-dimensional deconvolution as the imaging condition to further reduce the artifacts.

\section{SYNTHETIC EXAMPLES}

Here, we present two synthetic data results to demonstrate the effectiveness of Autofocus Imaging.

\section{Layer-cake model}

This subsurface model comprises a constant velocity and a vertically varying density profile (Figure $2 \mathrm{a}$ ). An absorbing boundary is used for the surface to ensure that no surface multiples are present in the data. The data acquisition is a fixed surface spread with the sources and receivers at $z=0 \mathrm{~m}$ and at regular lateral spacing of $10 \mathrm{~m}$. In addition to deconvolving the source signature from the surface reflection data, the direct arrivals were muted from the shot gathers. Beside this, no other processing was performed on the data; the data contain all orders of internal multiples. The images resulting from Autofocus Imaging are shown in Figure 3a and 3b. For comparison, the RTM image is shown in Figure 3c. Note that the internal multiples result in spurious reflectors in the RTM image, whereas they are absent in the image from Autofocus Imaging (Figure 3a). The Autofocus Imaging result produced using the deconvolution imaging condition (Claerbout, 1985) in Figure 3b still has minor artifacts, which can be further reduced by opting for a larger number of iterations in the imaging algorithm. On the other hand, the image resulting from crosscorrelation imaging condition (Figure 3a) is nearly devoid of these artifacts. As expected, the relative reflection amplitudes are better preserved with the deconvolution imaging condition as compared to the crosscorrelation imaging condition.

\section{Sigsbee model}

This is a variable-velocity and constant-density synthetic model. The primary challenge in this model is the poor subsalt image and the imaging of the salt-body-generated internal multiples. We use a portion of the Sigsbee $2 b$ model (Figure $4 a$ ) in this work to reduce the computational cost. As above, surface-related multiples are not generated by using an absorbing boundary at the surface. Similar to the acquisition for the layer-cake model, the data here are acquired on a fixed spread with the sources and receivers on the surface at regular lateral spacing of $25 \mathrm{~m}$.

Imaging is done using a smoothed velocity field as shown in Figure $4 \mathrm{~b}$; i.e., the velocity model in Figure $4 \mathrm{~b}$ is used in generating the direct arrivals $u^{i 0}$ in Autofocus Imaging and for generating the forward- and back-propagated wavefields in RTM. The results of Autofocus Imaging and RTM are shown in Figure 5a and 5b, respectively. For better clarity, zoomed portions of the images underneath the left salt flank are shown in Figure $6 \mathrm{a}$ and $6 \mathrm{~b}$. Note the presence of artifacts (pointed by arrows) below the salt body resulting from RTM (Figure 6b). Such artifacts could be interpreted as real subsurface structures and also can mask the true structure. Autofocus Imaging (Figures 5a and 6a), on the other hand, has imaged most of the internal multiples accurately. Increasing the number of iterations can further improve the quality of the image obtained from Autofocus Imaging at the expense of more computational time.

\section{ADVANTAGES}

There are multiple advantages of Autofocus Imaging over other imaging algorithms:

\section{Multiples are imaged}

As mentioned above, because the wavefields in Autofocus Imaging are reconstructed accurately, the image should be more accurate 
than those generated using existing imaging algorithms. Also, many orders of multiples are reconstructed and imaged accurately. Note that the internal multiples do not result in any spurious events in the image in Figure 5a but the RTM image (Figure 5b) contains multiple spurious reflectors. Imaging of internal multiples also renders their prediction and suppression unnecessary.

\section{Accurate amplitude}

Autofocus Imaging is based on inverse scattering theory and therefore the reconstructed wavefield in the interior of the medium is fairly accurate irrespective of the velocity and density distributions in the subsurface. This is because the wavefields are reconstructed using the reflection data that have all the amplitude effects in it (ignoring the effect of attenuation). Hence, the image resulting from Autofocus Imaging could potentially be closer to the true reflectivity of the subsurface (especially when using the deconvolution imaging condition). It might be possible to generate angle gathers for Autofocus Imaging in the same way as in RTM. This topic is currently under investigation. Amplitude variation with angle (AVA) analysis might also be more reliable because the wavefields are accurate.

\section{Targeted-oriented imaging}

Note that for the computation of the image at any location in the image space, we need the direct arrivals at the surface for an impulse at the image point. Because the computation of the direct arrivals can be done independently for each image point, it is possible to perform targeted-oriented imaging using Autofocus Imaging.

\section{Highly parallelizable}

Because the image at each location in the image space can be computed independently, the algorithm is highly parallelizable in the image space. For example, if there are $m$ grid points in the image space, $m$ processes could be run simultaneously on a cluster to obtain the image.
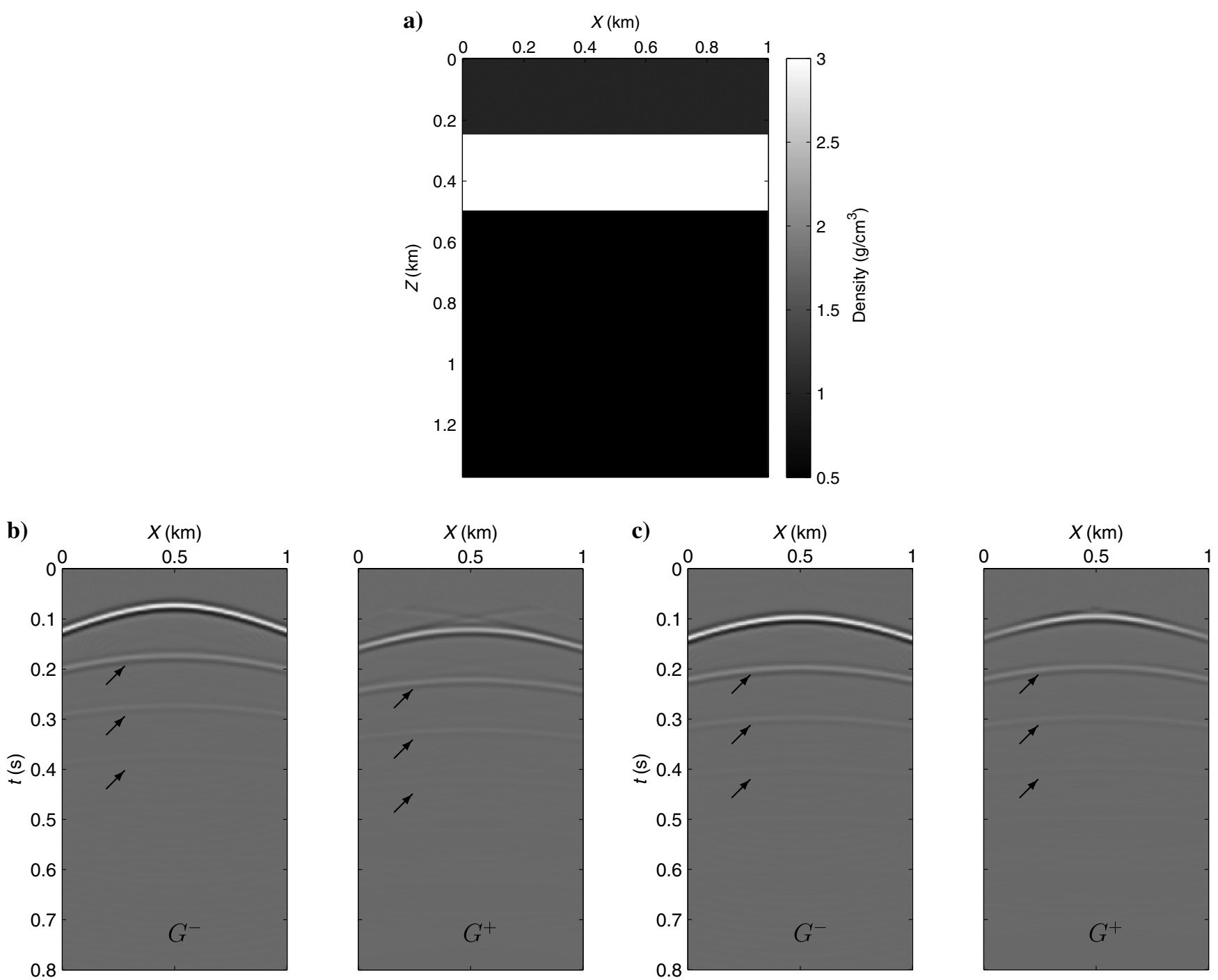

Figure 2. (a) Layer-cake subsurface density model. The velocity is uniform and equal to $5000 \mathrm{~m} / \mathrm{s}$. (b) The up- and down-going $G^{-}$and $G^{+}$ wavefields at a depth devoid of reflectors $(z=375 \mathrm{~m})$ and $(\mathrm{c})$ coinciding with a reflector $(z=500 \mathrm{~m})$. The arrows point to parts of the wavefield generated from internal multiples. 


\section{DISCUSSION}

\section{Acquisition design}

Implementation of Autofocus Imaging requires the generation of special shotpoint gathers, which we call fixed-spread gathers. These special gathers are such that each bin is a shot-gather comprising receivers at every bin location. For example, if the survey is divided into $n$ bins, then the sorted data for Autofocus Imaging comprise $n$ shot gathers with each shot gather containing $n$ traces. In other words, the resulting data are as if they were acquired on a fixed receiver-spread with every receiver location also being a shot location. However, it is not common practice to acquire data on such fixed spreads. Under such circumstances, conventional reflection seismic data (e.g., end-on 2D marine acquisition shown in a)

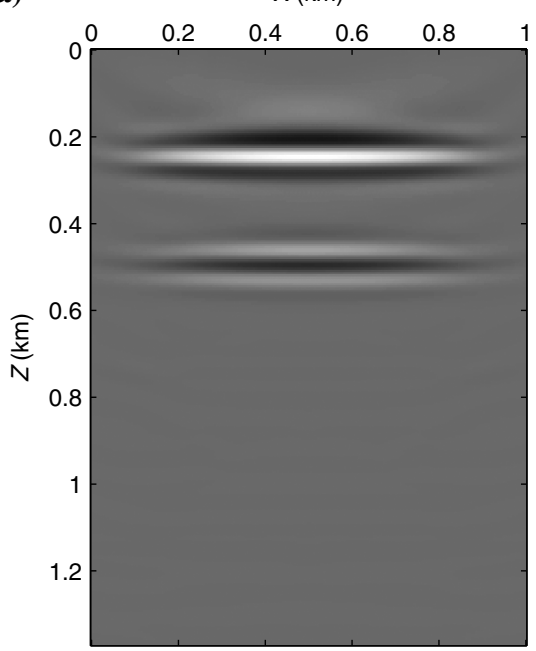

b)

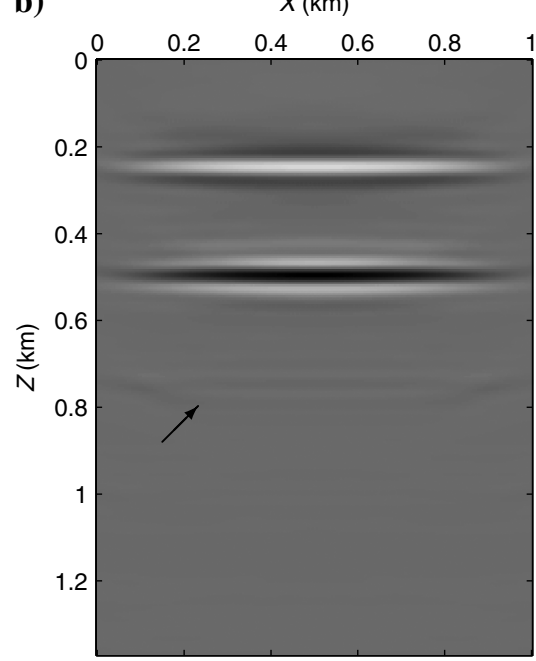

c)

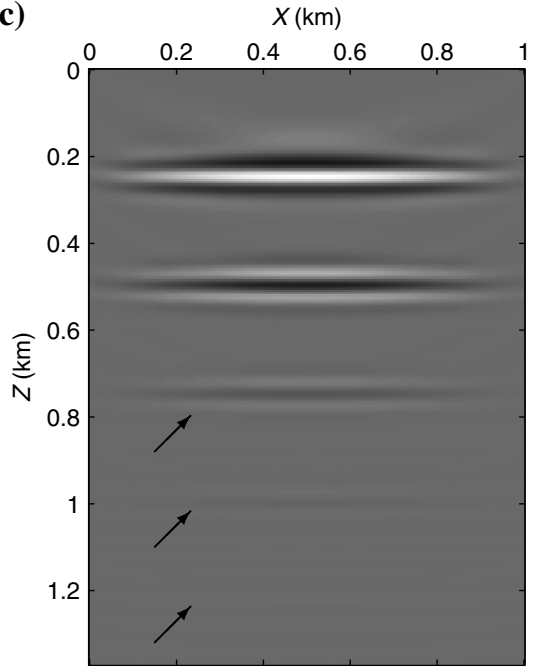

Figure 3. The image of the layer-cake model in Figure 2a resulting from (a) and (b) Autofocus Imaging and (c) RTM. The crosscorrelation and deconvolution imaging conditions are used in (a) and (b), respsectively. A constant velocity of $5000 \mathrm{~m} / \mathrm{s}$ was used for modeling and imaging. The Autofocus Imaging result is from $G^{+}$and $G^{-}$produced after 10 iterations. Imaging artifacts are denoted by arrows.

a)

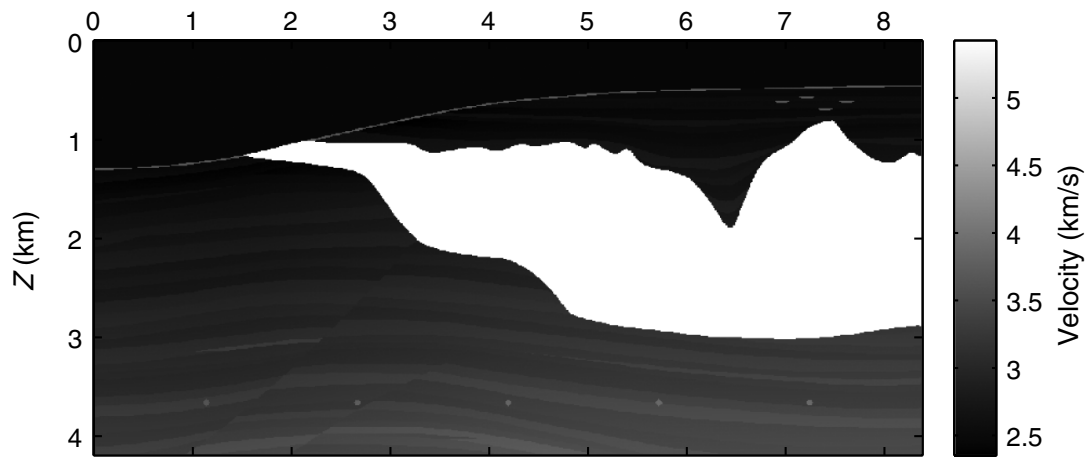

b)

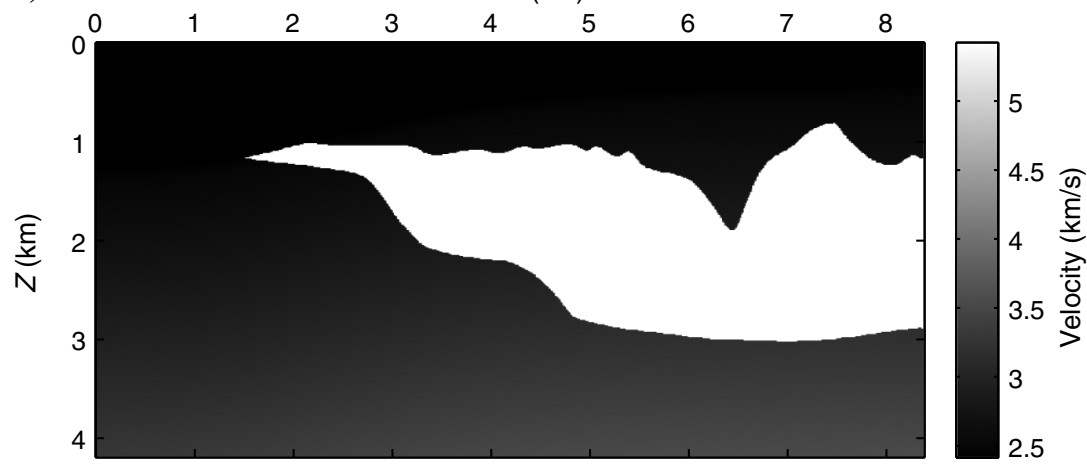

Figure 4. (a) The original Sigsbee velocity model and (b) its smoothed version used for imaging. A constant density of $1 \mathrm{~g} / \mathrm{cm}^{3}$ was used in generating the reflection data. 
Figure 5. Images of the Sigsbee model obtained from (a) Autofocus Imaging and (b) RTM. The autofocus image is generated with 20 iterations. The crosscorrelation imaging condition is used for generating both images.

a)

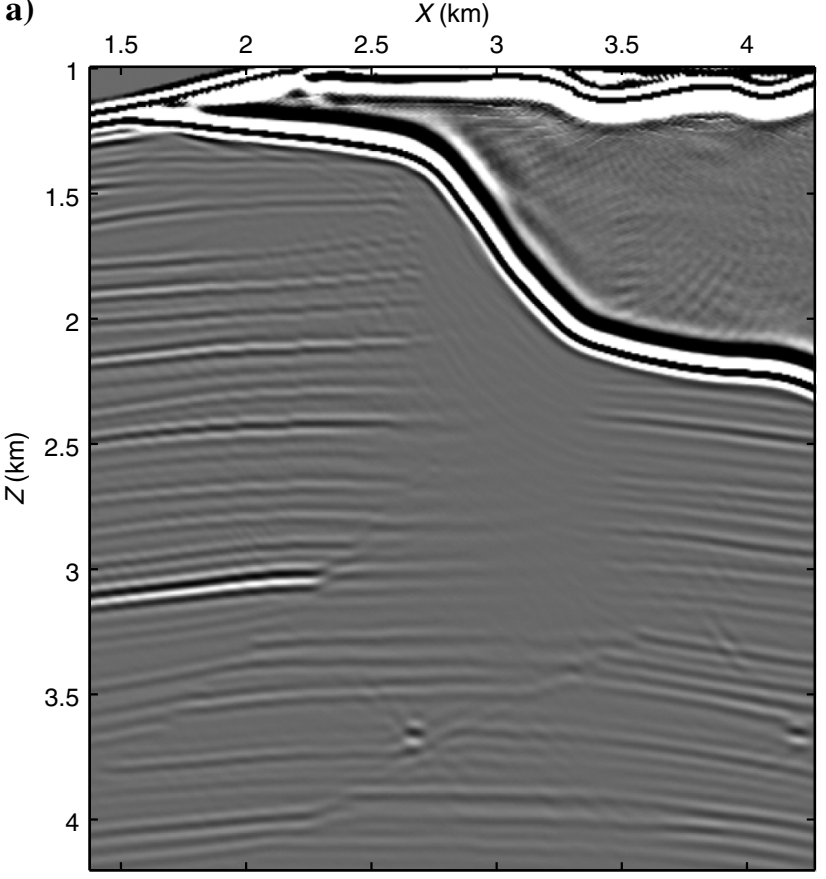

a)

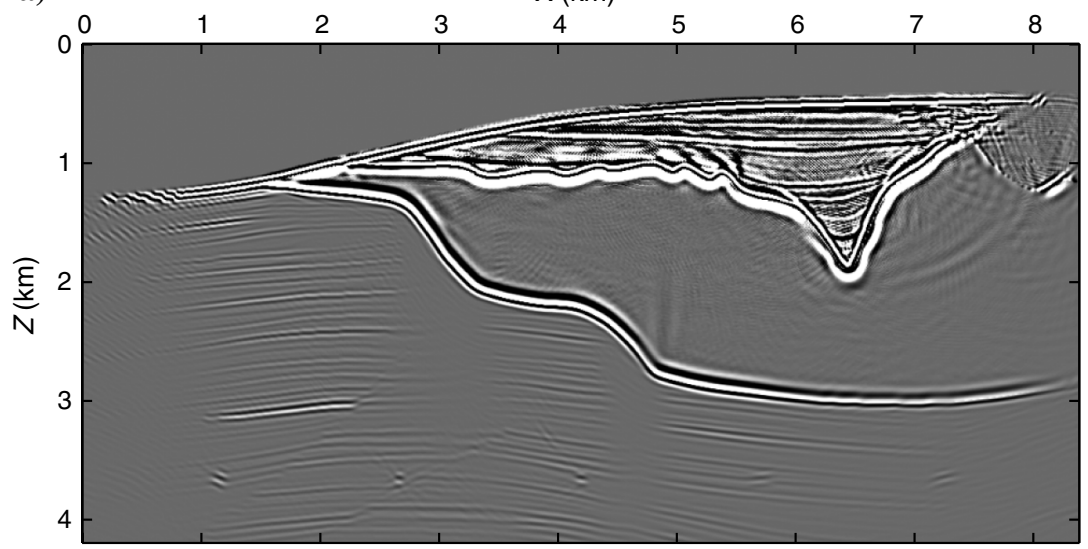

b)
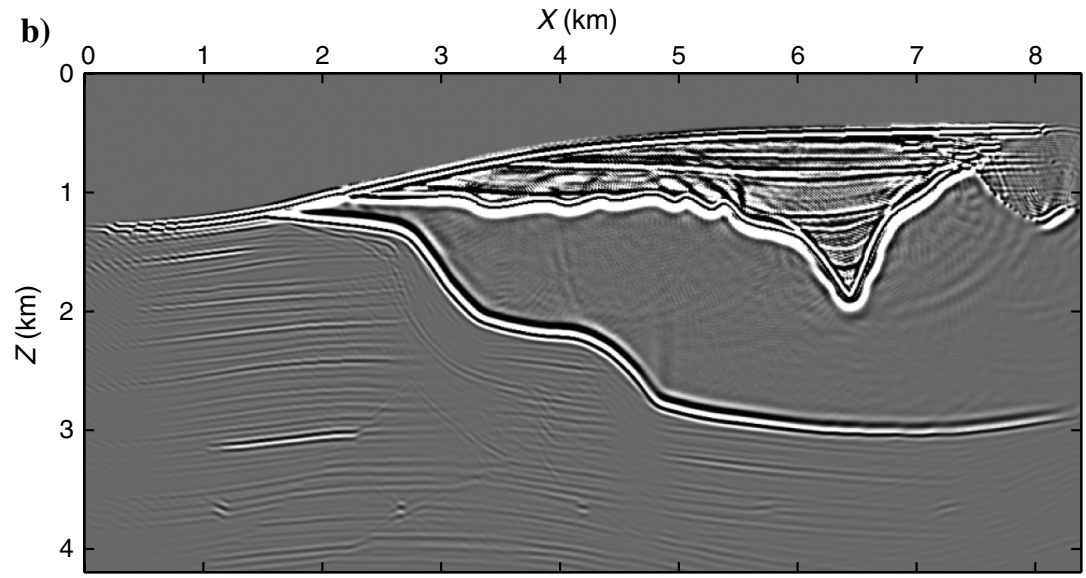

Figure 6. Zoomed portion of the subsalt images in Figure 5 of the Sigsbee model obtained using (a) Autofocus Imaging and (b) RTM. Imaging artifacts are denoted by arrows.

b)

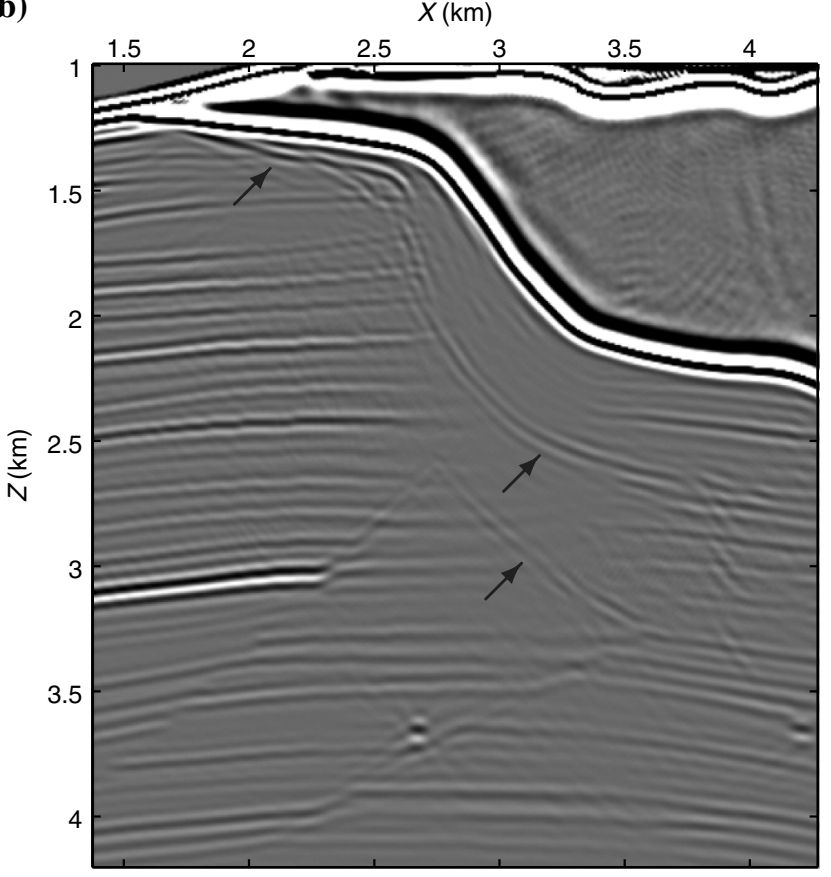


Figure 7a) can be sorted to generate the fixed-spread gathers (Figure 7b). Besides using conventional shot gathers in their construction, reciprocity can be used to further populate the gathers. If no real traces exist for the shot and receiver combination, the trace can be interpolated or assigned a null value. This is evident in Figure 7, in which some of the missing traces in Figure 7a have been populated using reciprocity (Figure $7 b$ ). Although many traces have been populated using reciprocity, other locations where no traces exist have been assigned a null value (white triangles in Figure $7 b$ ).

\section{Computation of direct arrivals}

The direct arrivals at the surface from an impulse at any image point can be computed either using ray tracing or solving the wave equation numerically using finite differences using a smooth background model for the velocity. If the background velocity field results in multipathing, one must make sure that the incident wavefield contains triplications; if not, the incident wave would not produce a quality focus at the image point. For a relatively uncomplicated subsurface, ray tracing should suffice. The numerical

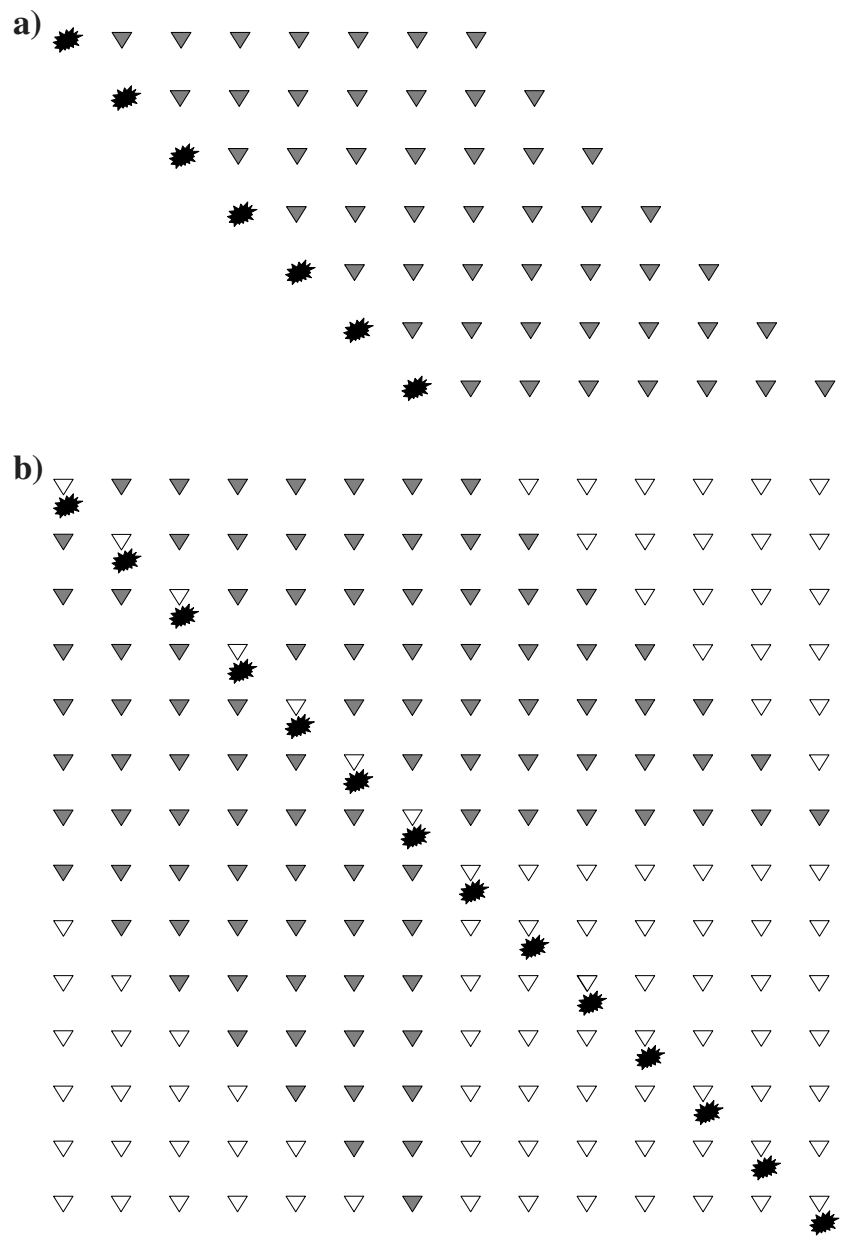

Figure 7. (a) Conventional end-on 2D seismic acquisition. The shots are represented by the explosive signs and the receivers by the triangles. (b) Fixed-spread acquisition obtained from the above end-on acquisition in (a). Reciprocity has been used to populate many traces. The null or dead traces are represented by white triangles. solution of the wave equation might be necessary for more intricate direct arrivals. Ray tracing, however, has one significant advantage: it is substantially cheaper than solving the wave equation numerically (especially in anisotropic media). This approach will also help us to image even high frequencies at no additional cost as opposed to other imaging algorithms, such as RTM, in which the computational cost increases with frequency.

\section{Computational expense}

The primary drivers of computational expense arise from the computation of direct arrivals and the many multidimensional convolutions involved in the iterative scheme. If the direct arrivals are computed using finite differences, then Autofocus Imaging is substantially more computationally intensive than RTM. There are two strategies for computing the direct arrivals between the image points and the surface receivers. First, the direct arrivals for each image point can be computed on the fly while the code is being executed. The number of forward computations will equal the number of image points. This will require significant processor power but have minimal disk input-output costs. Second, because there are far fewer receivers than image points, one could invoke reciprocity and compute the direct arrivals for all image points from each receiver location independently and then store them to the disk. Thus the number of forward computations will equal the number of receivers. Because of reciprocity, one can then read the direct arrivals from the disk for each image point; this, however, involves substantial disk input-output operations. Based on the number of receivers versus the number of image points, one can choose either of the above two strategies.

Also, the computational expense increases with the number of iterations used because of the multidimensional convolutions between the incident waves and the reflection response. More iterations are required for stronger internal multiples; for weak internal multiples, a few iterations will suffice.

\section{Source signature}

The autofocusing algorithm requires the Green's function at the surface, which is the reflection data corresponding to an input spike. The reflection data acquired in the field, however, are the Green's function convolved with the source signature. Therefore, it is critical to know the source signature in the reflection data. Among other methods (e.g., Robinson and Treitel, 1980), the method of Virtual Real Source (Behura and Snieder, 2013) yields accurate source signatures even if they vary among shots. Also, the adaptive surface-related multiple elimination (Verschuur et al., 1992) yields a reasonably accurate estimate of the source signature.

\section{CONCLUSIONS}

Autofocus Imaging, which is based on inverse scattering theory, shows promise in imaging complicated subsurfaces. Beside primaries, it can image internal multiples accurately, thereby eliminating most of the spurious events. This should make Autofocus Imaging useful for imaging poorly illuminated areas, especially underneath salt bodies. In addition to the above advantages, Autofocus Imaging can be used for targeted imaging, and should generate images and angle gathers with more accurate amplitudes. 


\section{ACKNOWLEDGMENTS}

We appreciate J. Thorbecke's help in implementation of the modeling code. Discussions with S. Singh, C. Fleury, F. Broggini, and F. Forghani were very useful. Reviews by J. Ajo-Franklin, M. Ravasi, and an anonymous reviewer helped polish the manuscript. Support for this work was provided by the Consortium Project on Seismic Inverse Methods for Complex Structures at Center for Wave Phenomena.

\section{REFERENCES}

Agranovich, Z. S., and V. A. Marchenko, 1963, The inverse problem of scattering theory, 1st ed.: Gordon and Breach.

Aktosun, T., and J. H. Rose, 2002, Wave focusing on the line: Journal of Mathematical Physics, 43, 3717-3745, doi: 10.1063/1.1483894.

Behura, J., and R. Snieder, 2013, Virtual real source: Source signature estimation using seismic interferometry: Geophysics, 78, no. 5, Q57-Q68, doi: 10.1190/geo2013-0069.1.

Behura, J., K. Wapenaar, and R. Snieder, 2012, Newton-Marchenko-Rose imaging: 82nd Annual International Meeting, SEG, Expanded Abstracts, doi: $10.1190 /$ segam2012-1531.1.

Broggini, F., and R. Snieder, 2012, Connection of scattering principles: A visual and mathematical tour: European Journal of Physics, 33, 593-613, doi: 10.1088/0143-0807/33/3/593.
Broggini, F., R. Snieder, and K. Wapenaar, 2013, Data-driven wavefield focusing and imaging with multidimensional deconvolution: Numerical examples for reflection data with internal multiples: Geophysics, 79, this issue, doi: 10.1190/GEO2013-0307.1.

Claerbout, J. F., 1985, Imaging the earth's interior: Blackwell Scientific Publications.

Marchenko, V. A., 1955, Reconstruction of the potential energy from the phase of scattered waves: Doklady Akademii Nauk SSSR, 104, 695698 (in Russian).

Robinson, E. A., and S. Treitel, 1980, Geophysical signal analysis: PrenticeHall Inc.

Rose, J. H., 2002a, Time reversal, focusing and exact inverse scattering, in Fink, M., W. A. Kuperman, J.- P. Montagner, and A. Tourin, eds., Imaging of complex media with acoustic and seismic waves 1st ed.: SpringerVerlag, 97-105.

Rose, J. H., 2002b, Single-sided autofocusing of sound in layered materials: Inverse Problems, 18, 1923-1934, doi: 10.1088/0266-5611/18/6/329.

Verschuur, D. J., A. J. Berkhout, and C. P. A. Wapenaar, 1992, Adaptive surface-related multiple elimination: Geophysics, 57, 1166-1177, doi 10.1190/1.1443330.

Wapenaar, K., F. Broggini, E. Slob, and R. Snieder, 2013, Three-dimensional single-sided Marchenko inverse scattering, data-driven focusing, Green's function retrieval, and their mutual relations: Physical Review Letters, 110, 084301, doi: 10.1103/PhysRevLett.110.084301.

Wapenaar, K., F. Broggini, and R. Snieder, 2011, A proposal for modelindependent 3D wave field reconstruction from reflection data: $81 \mathrm{st}$ Annual International Meeting, SEG, Expanded Abstracts, 3788-3792. 